\title{
THE NUMBER OF CAYLEY INTEGERS OF GIVEN NORM
}

\author{
by P. J. C. LAMONT
}

(Received 22nd September 1980)

Using results obtained by J. W. L. Glaisher $[1,2]$ for the number of representations $R_{r, s}(n)$ of $n$ as a sum of $r$ odd and $s$ even squares, formulae are derived for the number of Cayley integers of given norm $n$ in certain orders $o$. When computer generating order elements of given norm, the formulae can be used to verify that all the required elements have been obtained.

Let $\mathscr{C}$ be the classical Cayley algebra defined over the rationals with basis $\left\{i_{s}\right\}_{0}^{7}$ where $\left\{i_{s}\right\}_{0}^{4}$ is a basis of a quaternion algebra $\mathscr{H}_{4}, i_{0}=1, i_{1} i_{2} i_{3}=-1, i_{1} i_{4}=i_{5}, i_{2} i_{4}=i_{6}$, and $i_{3} i_{4}=i_{7}$. For $\xi=\sum_{s=0}^{7} x_{s} i_{s}, \bar{\xi}=2 x_{0}-\xi$ is called the conjugate of $\xi$. The real part $R(\xi)$ of $\xi$ is $x_{0}$. For $\xi=\xi_{0}+\xi_{1} i_{4}$ and $\eta=\eta_{0}+\eta_{1} i_{4}$, where $\xi_{t}$ and $\eta_{t}$ belong to $\mathscr{H}_{4}$ for $t=0$ and 1 , multiplication is defined in $\mathscr{C}$ by

$$
\xi \eta=\xi_{0} \eta_{0}-\bar{\eta}_{1} \xi_{1}+\left(\eta_{1} \xi_{0}+\xi_{1} \bar{\eta}_{0}\right) i_{4}
$$

The norm $N \xi$ of $\xi$ is $\xi \bar{\xi}$. Hence for any $\xi$ of $\mathscr{C}$

$$
\xi^{2}-2 R(\xi) \xi+N \xi=0 .
$$

We recall the following theorem. [4, Theorem 2.1].

(3) A non-rational Cayley number $\rho$ induces an automorphism $\xi \rightarrow \rho \xi \rho^{-1}$ of $\mathscr{C}$ if and only if

$$
4 R^{2}(\rho)=N \rho
$$

A set $o$ of Cayley numbers is called an order if, for any element $\xi$ of $o$, (2) has rational integral coefficients, and $o$ is closed under addition and multiplication. Elements of an order a are called Cayley integers.

Let $J$ be the order of $\mathscr{C}$ spanned by $\left\{i_{s}\right\}_{0}^{7}$ over $Z$. Let $J_{t}$ be obtained by adjoining

$$
\rho_{t}=\frac{1}{2}\left(1+u_{1}+u_{2}+u_{3}\right)
$$

to $J$, where $\left\{u_{s}\right\}_{1}^{3}$ is a multiplicatively associative set of distinct elements of $\left\{i_{s}\right\}_{1}^{7}$ such that $u_{1} u_{2} u_{3}=-1$, and $\left\{1, u_{1}, u_{2}, u_{3}, i_{t}, u_{1} i_{t}, u_{2} i_{t}, u_{3} i_{t}\right\}$ is the basis $\left\{i_{s}\right\}_{0}^{7}$ in some order. The set $\left\{u_{s}\right\}_{1}^{3}$ determines and is uniquely determined by $i_{t}$. For $1 \leqq t \leqq 7, J_{t}$ is an order of $\mathscr{C}$. Define $J_{0}$ to be the intersection of the seven orders $J_{t}$. For $\xi=\sum_{s=0}^{7} x_{s} i_{s}$, an element of $J_{0}$, the $x_{s}$ are either all integers or all half odd integers. Let a submodule $E$ of $J_{0}$ be 
defined by the condition:

$$
\xi \in E \text { if and only if } \xi \in J_{0} \text { and } \sum_{s=0}^{7} x_{s} \in 2 Z \text {. }
$$

All elements of $E$ have even norm. $J_{0}$ and $E$ are orders of $\mathscr{C}$.

Let $i, u, v, w$ be distinct elements of $\left\{i_{s}\right\}_{1}^{7}$ such that $i=u(v w)$. The mapping $\xi \rightarrow \rho \xi \rho^{-1}$ where

$$
\rho=\frac{1}{2}(1+u+v+w)
$$

applied to $\left\{i_{s}\right\}_{0}^{7}$ gives a new basis $\left\{e_{s}\right\}_{0}^{\gamma}$ of $\mathscr{C}$ that reproduces the multiplication table of the first basis. Let $J_{i}$ be the order obtained by adjoining $\left\{e_{s}\right\}_{1}^{7}$ to $J . J_{i}$ is independent of the choice of $u, v, w$ for which $u(v w)=i$ and is one of seven isomorphic maximal orders. The orders are obtained by letting $i$ take any value from the set $\left\{i_{s}\right\}_{1}^{7}$. For $i=i_{s}$, we write $J_{i}=M_{s}$. Each $M_{s}$ contains fourteen distinct sets of elements of the form $\sum_{r=1}^{4} x_{r} v_{r}$ where the $x_{r}$ are half odd integers. The $v_{r}$ take fourteen sets of values from $\left\{i_{s}\right\}_{0}^{7}$.

Let $a$ be any one of the orders $J, J_{0}, E, J_{s}$, and $M_{s}(1 \leqq s \leqq 7)$. Let $r_{s}(n)$ be the number of Cayley integers of norm $n$ in $\sigma$. Let $T=\sum_{m=0}^{\alpha} 2^{3 m}$ where $2^{\alpha}$ is the highest power of 2 dividing $n$. The formulae listed below hold when summation is taken over all indicated rational integral divisors of the integer $n$.

$$
\begin{gathered}
r_{J}(n)=r_{J}(1) \sum_{d \mid n}(-1)^{n+d} d^{3} \\
r_{J_{0}}(n)=r_{J_{0}}(1) \sum_{d \mid n} d^{3}, \quad \text { if } n \text { is odd. } \\
r_{J_{0}}(2 n)=r_{J_{0}}(1)(1+22 T)\left(\sum_{\substack{d \mid n \\
d \text { odd }}} d^{3}\right) . \\
r_{E}(2 n)=r_{E}(2) \sum_{d \mid n} d^{3}
\end{gathered}
$$

For $0=J_{s}$ or $M_{s}$

$$
\begin{gathered}
r_{o}(n)=r_{o}(1) \sum_{d \mid n} d^{3}, \quad \text { if } n \text { is odd. } \\
r_{J_{s}}(2 n)=r_{J_{s}}(1)(1+12 T)\left(\sum_{\substack{d \mid n \\
d \text { odd }}} d^{3}\right) . \\
r_{M_{s}}(2 n)=r_{M_{s}}(1) \sum_{d \mid 2 n} d^{3} . \\
r_{E}(2 n)=r_{M_{s}}(n) .
\end{gathered}
$$

It can be verified that $r_{J}(1)=r_{J_{0}}(1)=16, r_{J_{s}}(1)=48$, and $r_{M_{s}}(1)=r_{E}(2)=240$. The formulae (5) and (8) are known $[3,6]$. 
We outline the proof of (10). From (5),

$$
\begin{aligned}
r_{J}(2 n) & =16 \sum_{d \mid n}(-1)^{d} d^{3} \\
& =16\left(T-2+2^{3 \alpha+3}\right)\left(\sum_{\substack{d \mid n \\
d \text { odd }}} d^{3}\right) .
\end{aligned}
$$

$R_{8,0}(8 n)$ gives the number of representations of $2 n$ by 8 half odd integers. From Glaisher [1],

$$
R_{8,0}(8 n)=16^{2} \Delta_{3}^{\prime}(n)=16^{2} \cdot 2^{3 \alpha}\left(\sum_{\substack{d \mid n \\ d \text { odd }}} d^{3}\right) .
$$

$R_{4,4}(8 n)$ gives the number of representations of $2 n$ by 4 half odd integers and 4 integers.

$$
\begin{aligned}
R_{4,4}(8 n) & =1120 \Delta_{3}^{\prime}(2 n) \\
& =1120 \cdot 2^{3 \alpha+3}\left(\sum_{\substack{d \mid n \\
d \text { odd }}} d^{3}\right) .
\end{aligned}
$$

$R_{4,4}(8 n) /\left({ }_{4}^{8}\right)$ gives the corresponding number of representations with the 4 half odd integers in fixed positions. $J_{s}$ admits 2 such sets of positions. Hence

$$
r_{J_{s}}(2 n)=16\left(T-2+5 \cdot 2^{3 \alpha+3}\right)\left(\sum_{\substack{d \mid n \\ d \text { odd }}} d^{3}\right) .
$$

The result (10) follows.

\section{REFERENCES}

1. J. W. L. Glaisher, On the numbers of representations of a number as a sum of $2 r$ squares, where $2 r$ does not exceed eighteen, Proc. London Math. Soc. (2) 5 (1907), 479-490.

2. J. W. L. GlaisheR, On the representations of a number as the sum of two, four, six, eight, ten, and twelve squares, Quarterly J. 38 (1907), 1-62.

3. G. H. Hardy and E. M. Wright, An Introduction to the Theory of Numbers (Fourth Edition, Oxford, 1960).

4. P. J. C. Lamont, Arithmetics in Cayley's Algebra, Proc. Glasgow Math. Assoc. 6 (1963), 99-106.

5. R. A. Rankin, A certain class of multiplicative functions, Duke Math. J. 13 (1946), 281-306.

6. J. P. Serre, Cours d'Arithmétique (Paris, 1970).

Department of Quantitative and Information Science

College of Business

WESTERN ILLINOIS UNIVERSITY

MACOMB

ILLINOIS 61455 